\title{
EFICACIA DE LA QUIMIOTERAPIA DE INDUCCIÓN EN EL TRATAMIENTO DEL CÁNCER DE CABEZA Y CUELLO EN EL HOSPITAL SAN JUAN DE DIOS, DEL AÑO 2010 AL 2013
}

Fernández Barrantes, Cristina ${ }^{1}$; Ugalde Rojas, Irene ${ }^{1}$; Forester Quesada, Mario ${ }^{2}$; Cordero García, Eugenia ${ }^{3}$ y Ramos Esquivel, Allan ${ }^{4}$

${ }^{1}$ Hospital San Juan de Dios, Servicio de Farmacia, San José, Costa Rica ${ }^{2}$ HSJD- UCR. Estudiante de Internado en Farmacia, San Pedro, Costa Rica ${ }^{3}$ Universidad de Costa Rica, Facultad de Farmacia, San Pedro, Costa Rica

${ }^{4}$ Hospital San Juan de Dios, Servicio de Oncología, San José, Costa Rica

Resumen: Objetivos: evaluar la efectividad de la quimioterapia de inducción en el tratamiento del cáncer de cabeza y cuello en el Hospital San Juan de Dios del 2010 al 2013.

Métodos: Los expedientes clínicos de los pacientes diagnosticados con cáncer de cabeza y cuello y tratados del año 2010 al 2014, fueron analizados de manera retrospectiva. Se analizó información demográfica, clínica y de tratamiento. Las tasas de supervivencia y sobrevida libre de progresión fueron calculadas por medio de gráficos de Kaplan-Meier.

La mediana de seguimiento para los 33 pacientes fue de 20 meses. Del total de los pacientes incluidos en el estudio, 7 pacientes (21\%) fueron tratados con quimioterapia de inducción mientras que 26 pacientes (79\%) recibieron quimioradioterapia como tratamiento definitivo.

Conclusión: La respuesta a los 6 meses refleja una respuesta completa del 79\% en ambos grupos, sin embargo, no existe una diferencia estadísticamente significativa en la supervivencia de los pacientes con tratamiento de inducción respecto a aquellos que no recibieron este tratamiento.

Palabras clave: tratamiento farmacológico, sobrevida, neoplasia. Fuente: BIREME.

Recibido: 15 Diciembre 2017. Aceptado: 27 Febrero 2018. Publicado: 25 Abril 2018.

Revista electrónica publicada por el Departamento de Farmacología de la Escuela de Medicina de la Universidad de Costa Rica, 2060 San José, Costa Rica. ${ }^{\circledR}$ All rights reserved. Licensed under a Creative Commons Unported License. 


\section{EFFECTIVENESS OF INDUCTION CHEMOTHERAPY IN THE TREATMENT OF HEAD AND NECK CANCER IN THE SAN JUAN DE DIOS HOSPITAL OF THE YEAR 2010 TO 2013}

Abstract: to evaluate the effectiveness of induction chemotherapy in the treatment of head and neck cancer in Hospital San Juan de Dios from 2010 to 2013.

Methods: Clinical records of patients diagnosed with head and neck cancer and treated from 2010 to 2014 were retrospectively analyzed. Demographic, clinical and treatment information was analyzed. Progressionfree survival and survival rates were calculated using Kaplan-Meier plots.

The median follow-up for the 33 patients was 20 months. Of the total patients included in the study, 7 patients (21\%) were treated with induction chemotherapy, while 26 patients (79\%) received chemoradiotherapy as a definitive treatment.

Conclusion: The response at 6 months reflects a complete response of $79 \%$ in both groups; however, there is no statistically significant difference in the survival of patients with induction therapy compared to those who did not receive this treatment.

Key words: drug therapy, survivorship, neoplasms. Source: BIREME.

\section{INTRODUCCION}

Los tumores de cabeza y cuello se desarrollan a partir de células escamosas que se encuentran en las membranas mucosas, en más del $80 \%$ de los casos, por lo que, es posible encontrar tumores en los senos paranasales, cavidad oral, nasofaringe, orofaringe, hipofaringe y laringe. Estos crecimientos celulares pueden tener diversas etiologías, pero se les asocia más a factores de riesgo como el uso concomitante de tabaco y alcohol e infecciones como el virus de papiloma humano y Epstein-Barr. Otros factores que también pueden causar esta patología son una mala higiene dental, malos hábitos alimenticios y reflujo gastroesofágico [1-5].

Se estima que, a nivel mundial, unos 650000 pacientes son diagnosticados con cáncer de cabeza y cuello al año. La tasa de mortalidad es de unos 220 000 muertes al año, lo que representa de un 40$50 \%$ de supervivencia luego de 5 años y con una media de supervivencia de menos de 1 año en pacientes con metástasis o recurrentes. Según la Agencia Internacional de Investigación del Cáncer (IARC) en Costa Rica, la incidencia en el año 2012 de cáncer de laringe, cavidad oral, nasofaringe y faringe fue de 265 casos con una mortalidad de un $53 \%$ aproximadamente $[6,7]$.

Actualmente la cirugía y la radioterapia son las terapias más utilizadas para el manejo de esta patología y el tratamiento con quimioterapia o terapias moleculares se utilizan solas o en conjunto con radioterapia. La quimioterapia se utiliza como tratamiento paliativo en enfermedad avanzada o de forma adyuvante con el fin de mejorar los índices curativos, a pesar de esto, no mejora la sobrevida global o la sobrevida libre de progresión. Con respecto a la terapia de inducción existe gran controversia y aún no se acepta de manera universal como tratamiento para esta patología $[5,8]$.

Revista electrónica publicada por el Departamento de Farmacología de la Escuela de Medicina de la Universidad de Costa Rica, 2060 San José, Costa Rica. ${ }^{\circledR}$ All rights reserved. Licensed under a Creative Commons Unported License. 
En los últimos años se han realizado estudios multicéntricos en Europa con el objetivo de evaluar la quimioterapia de inducción en estos tumores. Estos tratamientos mostraron una mejor sobrevida en los pacientes, con diferencias en cuanto a los efectos adversos y calidad de vida, de acuerdo a la terapia utilizada [8-11].

Por lo tanto la evaluación de la efectividad de la quimioterapia de inducción en el tratamiento del cáncer de cabeza y cuello en el Hospital San Juan de Dios del 2010 al 2013 cobra relevancia con el presente estudio.

\section{MÉTODOS Y MATERIALES}

La investigación es un estudio observacional de tipo retrospectivo, donde se incluyeron inicialmente la totalidad de los expedientes (57 pacientes) con diagnóstico de Cáncer de Cabeza y Cuello del Hospital San Juan de Dios (HSJD) en el periodo comprendido entre enero del 2010 y diciembre del 2013.

No hubo distinción en el rango de edad de los pacientes ni la etnia de los mismos y se incluyó en la búsqueda tanto a hombres como mujeres. Se excluyó a menores de edad (18 años). Los expedientes se solicitaron al departamento de archivo del hospital y se revisaron de marzo a mayo del 2016.

Para la recolección de los datos, se realizó una revisión de los expedientes clínicos de los pacientes que cumplen con los criterios de inclusión. No todos los expedientes estaban disponibles en el archivo del hospital (no se pudo localizar 24 expedientes).

A partir de los expedientes, se recolectó información correspondiente a variables epidemiológicas, clínicas e histopatológicas dentro de las cuales se incluyó: fecha de nacimiento, género, fecha de diagnóstico, fecha de inicio de QT inducción, fecha de inicio de RT y QT, fecha de progresión, fecha de inicio de tratamiento adyuvante, fecha de fallecimiento, localización anatómica del tumor, estadio de la enfermedad al ingreso del estudio, estado funcional del paciente o ECOG al diagnóstico de la enfermedad, terapias utilizadas para inducción y adyuvancia, respuesta a los 6 meses de iniciada la terapia de inducción y toxicidad reportada.

El seguimiento en los pacientes incluidos en el estudio se realizó hasta mayo 2016 (fecha de censura), o al momento del fallecimiento.

La información recolectada se procesó, digitalizó y manejó en bases de datos en archivos del paquete estadístico Excel. El análisis de Sobrevida Global y Sobrevida libre de progresión se hizo por medio de análisis estadísticos no paramétricos (método de Kaplan Meier). El análisis de estos datos se realizó por medio del paquete estadístico SPSS 19.0.

Para la ejecución de esta investigación se realizó la solicitud de exención al Comité Local de Bioética del HSJD (JOM004-2016) dado que cumplía la exención mencionada en el artículo 7 de la Ley de Investigación Biomédica por tratarse de una investigación de tipo retrospectiva.

\section{RESULTADOS}

Este estudio analizó los casos de 33 pacientes con diagnóstico de Cáncer de Cabeza y Cuello del Hospital San Juan de Dios de San José, Costa Rica del año 2010 al 2013, con una mediana de seguimiento de 20 meses. Ninguno de los pacientes presentó metástasis al momento del diagnóstico (Cuadro n. $\left.{ }^{\circ}\right)$.

Todos los pacientes fueron tratados con quimioradioterapia como tratamiento adyuvante. Sin embargo, de los 33 pacientes incluidos en el estudio, 7 pacientes (21\%) recibieron además, ciclos de quimioterapia de inducción con esquemas de cisplatino y fluorouracilo o cisplatino y paclitaxel y fluorouracilo (Cuadro n. ${ }^{\circ}$ ).

La media de días desde el momento del diagnóstico hasta en inicio de la terapia de inducción fue de 110.38 días mientras que el tiempo trascurrido para recibir la radioterapia fue de 4.74 meses (144.57 días). La respuesta que tuvieron los pacientes al

Revista electrónica publicada por el Departamento de Farmacología de la Escuela de Medicina de la Universidad de Costa Rica, 2060 San José, Costa Rica. ${ }^{\circledR}$ All rights reserved. Licensed under a Creative Commons Unported License. 
tratamiento, con o sin inducción, en un periodo de 6 meses se puede observar en el Cuadro $n .^{\circ} 3$.

Cuadro n. ${ }^{\circ}$ 1. Características demográficas de los pacientes incluidos en el estudio.

\begin{tabular}{|c|c|}
\hline Variable & Número (\%) \\
\hline Edad media (Años) & 56 \\
\hline Edad \pm & 21,03 \\
\hline \multicolumn{2}{|l|}{ Sexo } \\
\hline Femenino & $6(18.2)$ \\
\hline Masculino & $27(81.8)$ \\
\hline \multicolumn{2}{|l|}{$\mathrm{T}$} \\
\hline $\mathrm{Tx}$ & $4(12.1)$ \\
\hline $\mathrm{T} 1$ & $2(6.1)$ \\
\hline $\mathrm{T} 2$ & $8(24.2)$ \\
\hline T3 & $8(24.2)$ \\
\hline $\mathrm{T} 4$ & $11(33.3)$ \\
\hline \multicolumn{2}{|l|}{$\mathrm{N}$} \\
\hline 0 & $13(39.4)$ \\
\hline 1 & $7(21.2)$ \\
\hline 2 & $7(21.2)$ \\
\hline 3 & $5(15.2)$ \\
\hline 4 & $1(3.0)$ \\
\hline \multicolumn{2}{|l|}{ M } \\
\hline 0 & $33(100)$ \\
\hline \multicolumn{2}{|l|}{ ECOG } \\
\hline 0 & $30(90.9)$ \\
\hline 1 & $2(6.1)$ \\
\hline 2 & $1(3.0)$ \\
\hline \multicolumn{2}{|c|}{ Localización del Tumor } \\
\hline Nasofaringe & $13(39.3 \%)$ \\
\hline Hipofaringe & $3(9.1 \%)$ \\
\hline Laringe & $1(3.0 \%)$ \\
\hline Orofaringe & $12(36.4 \%)$ \\
\hline & $4(12.1 \%)$ \\
\hline
\end{tabular}

La Figura . $^{\circ} 1$ compara las tasas de recurrencia de los sujetos que recibieron inducción versus aquellos sin esta modalidad, con un valor de $\mathrm{p}=0.13$.

No hubo una diferencia estadísticamente significativa en la supervivencia de los pacientes y la sobrevida libre de progresión.

Finalmente los pacientes que fallecieron representan el $15.2 \%$ del total en estudio.

Cuadro n. ${ }^{\circ}$ 2. Tratamiento de inducción y de adyuvancia utilizado.

\begin{tabular}{ll}
\hline Tratamiento utilizado & n (\%) \\
\hline $\begin{array}{l}\text { Inducción: } \\
\quad \text { Cisplatino }\end{array}$ & $2(28)$ \\
Cisplatino + 5FU & $1(14)$ \\
Cisplatino + 5FU + Paclitaxel & $4(57)$ \\
\hline & \\
Adyuvancia: & $1(3)$ \\
RT + Cisplatino+ 5FU & $1(3)$ \\
RT + 5FU & $1(3)$ \\
RT + Carboplatino & $3(9.1)$ \\
RT + Cetuximab & $7(21.2)$ \\
RT & $20(66.7)$ \\
RT + Cisplatino & \\
\hline
\end{tabular}

Cuadro n. ${ }^{\circ}$ 3. Evolución a 6 meses de los pacientes incluidos en el estudio.

\begin{tabular}{lcc} 
Tratamiento & $\begin{array}{c}\text { Respuesta } \\
\text { Completa (\%) } \\
\text { Sin }\end{array}$ & $\begin{array}{c}\text { Respuesta Parcial } \\
(\%)\end{array}$ \\
$\begin{array}{l}\text { Inducción } \\
\text { Con } \\
\text { Inducción }\end{array}$ & $5(80)$ & $5(20)$ \\
\hline
\end{tabular}

Revista electrónica publicada por el Departamento de Farmacología de la Escuela de Medicina de la Universidad de Costa Rica, 2060 San José, Costa Rica. ${ }^{\circledR}$ All rights reserved. Licensed under a Creative Commons Unported License. 
Figura n. ${ }^{\circ}$ 1. Probabilidad de sobrevida libre de recurrencia de los pacientes de acuerdo al uso de tratamiento de inducción más quimioradioterapia versus sólo quimioradioterapia.

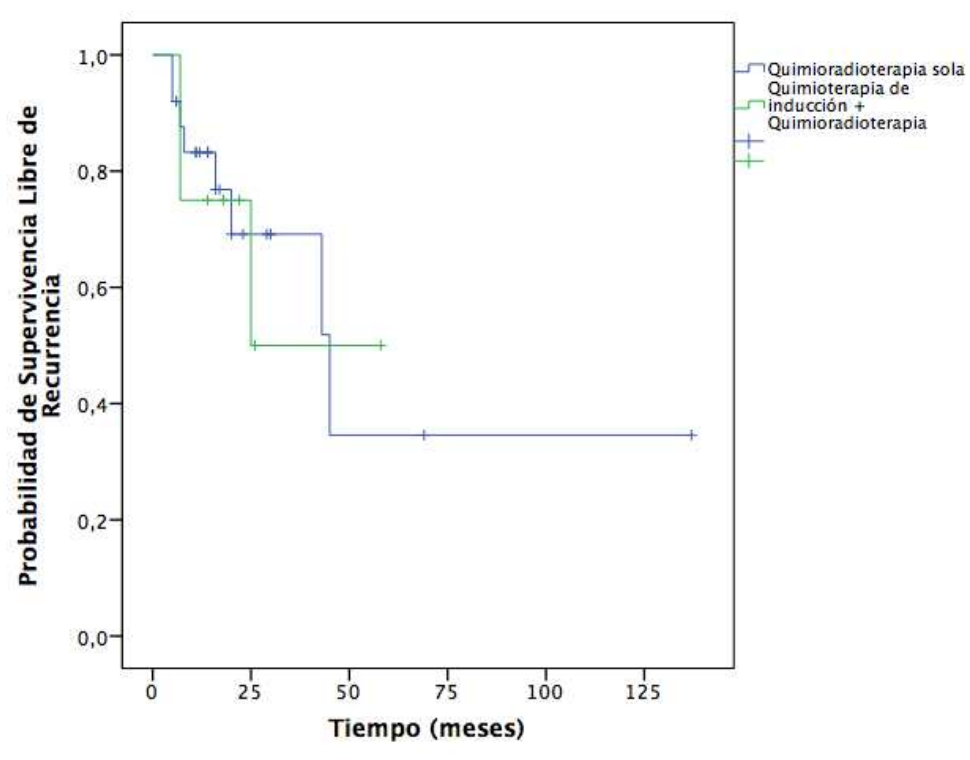

\section{DISCUSIÓN}

A pesar de que el tratamiento óptimo no quirúrgico para el carcinoma de cabeza y cuello es controversial, la quimioradioterapia es el estándar terapéutico para esta condición, en casos donde no sea posible la opción quirúrgica. Sin embargo, diversos estudios sugieren un aumento de la supervivencia del paciente y mejores tasas de respuesta a los esquemas quimioterapéuticos más modernos con el uso de quimioterapia de inducción respecto a la terapia estándar. Existen discrepancias respecto al aumento de la toxicidad y al impacto que esta pueda tener en la adherencia a la terapia definitiva [12].

La edad promedio en los pacientes seleccionados tuvo una media de 56 años, este dato según la literatura es muy variable ya que depende de la población que se engloba en el estudio. Esta variable no es generalmente reconocida como un factor determinante en el desenlace de la enfermedad en pacientes con esta patología, sin embargo, diversos meta-análisis sugieren un mejor beneficio de la quimioterapia en pacientes jóvenes con tumores locales avanzados [13].

Otro aspecto a discutir es el sexo, en vista de que en la literatura encontrada, las muestras poblacionales utilizadas denotan un mayor número de hombres respecto a las mujeres lo cual sugiere una mayor incidencia de este tipo de patologías en el sexo masculino. En el presente estudio, hubo una mayoría de pacientes masculinos tal como señala la literatura [1416].La estadificación en este estudio tiene similitudes y discrepancias respecto a lo establecido en diversos estudios y meta-análisis en donde la mayor cantidad de pacientes presentan un tumor con invasión de estructuras adyacentes profundas (T4) y una invasión a ganglios linfáticos categorías N1 y N2 lo cual concuerda con los resultados obtenidos. Finalmente, para este estudio no se utilizaron pacientes con metástasis a otros órganos y los valores de ECOG de la gran mayoría fueron de 0 o 1 lo que permite una mejor tolerancia a la terapia [14-17].

En este estudio, el mayor porcentaje de casos corresponden a pacientes con tumores a nivel de cavidad oral y laringe, sin embargo, en la literatura se reportan una mayoría de pacientes con cáncer en la zona orofaríngea. Esto se puede deber a la poca cantidad de pacientes que se pudo incluir en el estudio, en donde no se refleje la incidencia real de la enfermedad en nuestro país $[6,13,14,16]$.

Respecto a la terapia utilizada, en los pacientes estudiados la inducción con Paclitaxel, 5-FU y Cisplatino fue la más frecuente seguido de Cisplatino y 5-FU. Lo anterior responde a grandes estudios realizados, como el TAX 323 y TAX324, en donde se comparó la respuesta de los pacientes a los dos esquemas de tratamiento anteriormente mencionados con el fin de evaluar el beneficio de un esquema de respecto al otro. La supervivencia libre de progresión y el fallecimiento en pacientes con cáncer hipo faríngeo y de laringe disminuyó de

Revista electrónica publicada por el Departamento de Farmacología de la Escuela de Medicina de la Universidad de Costa Rica, 2060 San José, Costa Rica. ${ }^{\circledR}$ All rights reserved. Licensed under a Creative Commons Unported License. 
manera significativa en pacientes con tratamiento de inducción con Paclitaxel, Cisplatino y 5-FU con una extensión de 2.8 meses en la progresión de la enfermedad de los pacientes y 4.3 meses en la mediana de supervivencia de los pacientes respecto a los pacientes que fueron tratados con cisplatino y 5 -FU $[13,18,19]$.

Asimismo, el tratamiento adyuvante más utilizado fue la combinación de RT con Cisplatino, lo cual concuerda con lo establecido en diversos estudios como el de Hu-Ming, Hitt, Takacsi-Nagy y Ghi. No obstante, existen otros que validan el uso de radioterapia como tratamiento base, como lo establecido por Al-Wassia en su estudio en Arabia Saudita. Esto refleja la diversidad de esquemas terapéuticos que se pueden realizar en los pacientes con el fin de paliar o curar la patología de fondo [14$16,19]$.

La evolución que tuvieron los pacientes en este estudio es de carácter variable respecto a lo reportado en los estudios de Al-Wassia y Hu-Ming. En el primero la mayoría de pacientes tuvo una respuesta completa mientras que en el segundo estudio la respuesta parcial fue el común denominador en los pacientes tratados. En nuestro estudio $26(78 \%)$ pacientes tuvieron una respuesta completa a la terapia que se les brindo en la institución. Cabe destacar que del total de esos pacientes 5 de ellos recibieron quimioterapia de inducción $[14,15]$.

Las tasas de supervivencia libre de recurrencia en el tiempo, presentan una mediana de 45 meses desde el momento del diagnóstico hasta la reaparición del tumor con un total de 16 censuras en donde no hubo recurrencia. Además, la recurrencia que tuvieron los pacientes con quimioterapia de inducción versus los que no se sometieron a esta fue muy similar lo cual refleja que no hay diferencia estadísticamente significativa en la supervivencia y la sobrevida libre de progresión con el uso de ambos tratamientos. Lo anterior concuerda con diversos estudios revisados en un meta-análisis realizado por Budach et al. y la información recopilada por Hu-Ming y Al-Wassia, en donde no existe beneficio del uso de la quimioterapia de inducción en cáncer de cabeza y cuello [14-16].

Un planteamiento a la anterior problemática es la baja tolerancia a la quimioradioterapia después de una exposición a ciclos de tratamiento de inducción. Varios estudios como el PARADIGM mostraron pobre adherencia en pacientes que recibieron inducción o inclusive que nunca comenzaron la quimioradioterapia definitiva. Aquellos sujetos que lograron llevar el tratamiento completo tuvieron una mayor incidencia de toxicidades, aumento en la debilidad corporal y una disminución en la tasa de supervivencia respecto a los que no llevaron la inducción [12].

Lo recabado con el presente estudio refleja lo que se ha venido dando en otras latitudes en donde a la quimioterapia de inducción no mejora la supervivencia del paciente a largo plazo, a pesar de que los esquemas con cisplatino, docetaxel y fluorouracilo demuestran menores toxicidades y mejores respuestas en pacientes con cáncer laríngeo e hipofaríngeo respecto a los tratamiento con cisplatino y fluorouracilo.

Estudios como el presente permiten delimitar y mejorar la efectividad de los tratamientos empleados a nivel nacional mediante la obtención de información en un medio local y así validar el accionar a nivel institucional y nacional.

Las limitaciones más importantes para el presente estudio fueron la cantidad de expedientes que se lograron revisar. De haber sido una población más grande los resultados hubieran sido más representativos, el sesgo sería menor y se reflejaría de mejor manera la realidad que se vive en el Hospital San Juan de Dios.

\section{CONCLUSIONES}

- La quimioterapia de inducción para el tratamiento del cáncer de cabeza y cuello no mejora la supervivencia del paciente a largo plazo.

Revista electrónica publicada por el Departamento de Farmacología de la Escuela de Medicina de la Universidad de Costa Rica, 2060 San José, Costa Rica. ${ }^{\circledR}$ All rights reserved. Licensed under a Creative Commons Unported License. 
- El tratamiento adyuvante con RT y cisplatino es el más indicando en este tipo de pacientes, sin embargo existen otras alternativas para pacientes que no puedan tolerar esta combinación.

FUENTE DE FINANCIAMIENTO: Universidad de Costa Rica.

\section{REFERENCIAS}

1. Vokes E. Head and Neck Cancer. En: Harrison's Principles of Internal Medicine. Kasper D, Fauci A, Hauser S, Longo D, Jameson J, Loscalzo J (eds). McGraw-Hill Companies Inc, New York, United States. $19^{\text {th }}$ edition 2015, cap 106.

2. Weinberger P.M., Terris D.J. Otolaryngology: Head \& Neck Surgery. En: Current Diagnosis \& Treatment: Surgery. Doherty G.M. (Eds). McGrawHill Companies Inc, New York, United States. 14 edition 2015, cap 8.

3. Bussmann L, Busch C, Rieckmann T. Perspectives in chemosensitivity and chemoresistance assays and their implementation in head and neck cancer. European Archives of Othorhinolaryngology. 2016; 10:1007.

4. Rudin C, Marur S. Head and neck squamous cell carcinogenesis: Molecular and genetic alterations. Brockstein: 2016 Uptodate Topic 3387 Version 11.0 .

5. López J, Navarro E, Molina F, et al. Cáncer de cabeza y cuello. Medicine. 2013;11:1447-60.

6. Globocan 2012: Estimated Cancer Incidence, Mortality and Prevalence Worldwide in 2012. (Acceso 22 de Febrero de 2016).

7. Piedra, V. Manual de Normas para el Tratamiento de Cáncer en Costa Rica. 1era Ed. San José: Imprenta Nacional, 2014.

8. Gold K, Neskey M, William W. The Role of Systemic Treatment Before, During and After Definitive Treatment. Otolaryngologic Clinics of North America. 2013; 46(4): 645-656.

9. Vokes, E. Induction Chemotherapy for Head and Neck Cancer: Recent Data. The Oncologist. 2010;15(3): 3-7.
10. Hanna G, Haddad R, Lorch J. Induction Chemotherapy for Locoregionally Advanced Head and Neck Cancer: Past, Present, Future? The Oncologist. 2013;18: 288-293.

11. Wingard, J. Prophylaxis of infection during chemotherapy-induced neutropenia in high-risk adults. Marr: 2016. Uptodate Topic 1153 Version 25.0 .

12. Sher D, Shwartz D, Niedzi L, et al. Comparative effectiveness of induction chemotherapy for oropharyngealsquamous cell carcinoma: A population-based analysis. Oral Oncology. 2016; 54: 58-67.

13. Lorch J, Haddad R, Tan $M$, et al. Induction chemotherapy with cisplatin and fluorouracil alone or in combination with docetaxel in locally advanced squamous-cell cancer of the head and neck: long-term results of the TAX 324 randomized phase 3 trial. Lancet Oncology. 2011; 12: 153-59.

14. Hu-Ming W, Chien-Yu L, Shu-Nang $\mathrm{N}$, et al. Induction chemotherapy with dose-modified docetaxel, cisplatin, and 5-fluorouracil in Asian patients with borderline resectable or unresectable head and neck cancer. Journal of the Formosan Medical Association. 2017; 116: 185-192.

15. Al Wassia R, Abusanad A, Awad N, et al. Outcomes of Saudi Arabian Patients with Nasopharyngeal Cancer Treated with Primarily Neoadjuvant Chemotherapy Followed by Concurrent Chemoradiotherapy. Journal of Global Oncology. 2016; 2: 123-128.

16. Budach W, Kammers $\mathrm{K}$, Orth $\mathrm{K}$, et al. Induction chemotherapy followed by concurrent radiochemotherapy versus concurrent radiochemotherapy alone as treatment of locally advanced squamous cell carcinoma of the head and neck (HNSCC): A meta-analysis of randomized trials. Radiotherapy and Oncology. 2016;118: 238243.

17. Vermorken J, Remenar E, Degardin M, et al. Cisplatin, Fluorouracil, and Docetaxelin Unresectable Head and Neck Cancer. NEJM. 2007; 357:1695-704.

18. Posner M, Hershock D, Blajman, C, et al. Cisplatin and Fluorouracil Alone or with Docetaxel in Head and Neck Cancer. NEJM. 2007; 357:1705-15. 
19. National Comprehensive Cancer Network. NCCN Guidelines Version I. 2015. Head and Neck Cancers. (Último acceso el 18 de Junio de 2016). Disponible en:

https://www.nccn.org/professionals/physician_gls Lf guidelines nojava.asp.

\section{CORRESPONDENCIA}

Cordero García, Eugenia

eugenia.corderogarcia@ucr.ac.cr 